\title{
Heterogeneous mutation pattern in tumor tissue and circulating tumor DNA warrants parallel NGS panel testing
}

\author{
Qiaomei Guo ${ }^{1+}$, Junlei Wang ${ }^{2 \dagger} \mathbb{D}$, Jianfeng Xiao ${ }^{3}$, Lin Wang ${ }^{1}$, Xiaomeng Hu', Wenjun Yu', Gang Song ${ }^{3}$,
} Jiatao Lou ${ }^{1 *}$ and JianFeng Chen ${ }^{2^{*}}$ (D)

\begin{abstract}
Liquid biopsy by genotyping circulating tumor DNA (ctDNA) has provided a non-invasive approach in assessing tumor genomic alterations in clinical oncology. However, emerging evidence in clinical settings has shown significant discordance in the genomic alterations between matched tumor tissue and blood ctDNA samples, and even between the same set of blood samples analyzed on different testing platforms. Thus, it is necessary to study underlying causes of discrepancies in these studies by genotyping tumor tissue and ctDNA in parallel using next generation sequencing (NGS) panels based on the same technology. Here we enrolled 56 non-small-cell lung cancer (NSCLC) patients and evaluated tumor tissue genotyping and ctDNA based liquid biopsy by parallel NGS panel testing and compared different sample preparation conditions. Somatic mutations in plasma cell-free DNA (cfDNA) were detected in 63.6\% patients with earlystage NSCLC and $60 \%$ patients with advanced-stage NSCLC. The overall concordance between matched formalin-fixed paraffin-embedded sample and cfDNA was 54.6\% in early-stage NSCLC patients and 80\% in advanced-stage NSCLC patients. The positive concordance rate was $44.4 \%$ and $71.4 \%$ in early-stage and advanced-stage patients, respectively. Using fresh frozen tumor samples did not improve the overall concordance rate between matched tumor tissue and cfDNA. Processing blood samples beyond $4 \mathrm{~h}$ after blood draw significantly decreased the detection rate of somatic mutations in cfDNA. Thus, the concordance rate between tumor tissue-based and ctDNA-based genotyping in clinical samples can be affected by multiple pre-analytical, analytical and biologic factors. Parallel NGS panel testing on both sample types for each patient may be warranted for effective guidance of cancer targeted therapies and possible early detection of cancer.
\end{abstract}

Keywords: Liquid biopsy, Circulating tumor DNA, NGS,non-small-cell lung cancer, Somatic mutations, Concordance

\section{Main text}

Genotyping tumor tissue biopsy has become a standard practice in clinical oncology for cancer patient management. Recently, liquid biopsy using circulating tumor DNA (ctDNA) has provided a non-invasive approach in assessing tumor genomic alterations for cancer early detection, personalized therapy and treatment monitoring

\footnotetext{
* Correspondence: loujiatao@126.com; jfchen@sibcb.ac.cn

${ }^{\dagger}$ Qiaomei Guo and Junlei Wang contributed equally to this work.

'Department of Laboratory Medicine, Shanghai Chest Hospital, Shanghai Jiao Tong University, Shanghai, China

${ }^{2}$ State Key Laboratory of Cell Biology, CAS Center for Excellence in Molecular Cell Science, Shanghai Institute of Biochemistry and Cell Biology, Chinese Academy of Sciences, University of Chinese Academy of Sciences, 320 Yueyang Road, Shanghai 200031, China

Full list of author information is available at the end of the article
}

[1-3]. Commercially available tissue genotyping and liquid biopsy tests, including FoundationOne (F1), Guardant360 (G360) and PlasmaSELECT (PS), self-reported high accuracy, sensitivity and specificity to detect tumor-specific genomic alterations [4-6]. However, independent studies reported significant discordance in testing results between matched tumor tissues and ctDNA generated on F1 and G360 [7]. One recent study reports high discordance in ctDNA results for the same set of blood samples between G360 and PS [8]. The inaccurate genetic profiling in actual clinical settings has raised serious concerns about the risks of misguiding treatment decisions to cancer patients. In these studies, tissue and blood specimens were shipped to different vendors for DNA extraction, library preparations, targeted NGS and data analysis. The underlying causes of

(c) The Author(s). 2018 Open Access This article is distributed under the terms of the Creative Commons Attribution 4.0 International License (http://creativecommons.org/licenses/by/4.0/), which permits unrestricted use, distribution, and 
Table 1 Clinical characteristics of NSCLC patients with both tissue and ctDNA NGS testing

\begin{tabular}{lll}
\hline Characteristic & Number & Percentage(\%) \\
\hline Age, years & & \\
Mean (SD) & 59.73 & \\
Median (range) & $60(42-82)$ & \\
Gender & & \\
Female & 29 & 51.79 \\
Male & 27 & 48.21 \\
Stage & & \\
I & 38 & 67.86 \\
II & 7 & 12.50 \\
III & 9 & 16.07 \\
IV & 2 & 3.57 \\
Cytological diagnosis & & 82.14 \\
Adenocarcinoma & 46 & 17.86 \\
Squamous cell carcinoma & 10 & \\
\hline
\end{tabular}

discrepancies in these studies are difficult to be identified as the testing results were generated on clinical specimens across different testing platforms. Thus, genotyping tumor tissue and ctDNA in parallel on the same testing platform is important for clarifying this question prior to rigorous cross-platform comparisons using a large number of clinical samples.

To evaluate tumor tissue genotyping and ctDNAbased liquid biopsy by parallel NGS panel testing, we enrolled a total of 56 newly diagnosed early-stage (stages I and II) and advanced-stage (stages III and IV) non-small-cell lung cancer (NSCLC) patients (Table 1, and Additional file 1: Table S1). Blood samples from these patients were collected within 0-26 days before surgery. Each patient had matched formalin-fixed paraffin-embedded (FFPE) tumor tissue and germline DNA extracted from white blood cells. Matched fresh frozen (FF) tissue was also available for 21 out of 56 patients. We analyzed genomic alterations in cfDNA, matched germline, FFPE and FF DNA samples using NGS targeted sequencing panels. The Lung and Colon Cancer Panel (LC103) and the high sensitivity Lung Cancer Panel (L82) from Pillar Biosciences Inc. (Fig. 1 and Additional file 2) were used for tumor tissue and cfDNA samples, respectively. Different plasma sample processing time is also compared.

We examined somatic alterations in 21 matched NSCLC tumor biopsies and plasma cfDNA samples, which were obtained at the time of surgery and processed within $2 \mathrm{~h}$ after blood collection. Somatic mutations in cfDNA were detected in 7 out of 11 (63.6\%) early-stage NSCLC patients and 6 out of $10(60 \%)$ patients with advanced-stage NSCLC (Fig. 2a, b and Additional file 3: Table S2). In 14 low frequency cfDNA-specific alterations detected by $\mathrm{L} 82$ panel sequencing, 13 of them were also detected in cfDNA by droplet digital PCR (ddPCR) (Additional file 4: Table S3). No somatic variant was detectable in 5 patients ( 2 stage I and 3 stage III).

We further compared the concordance between FFPE tumor biopsy and ctDNA genomic profiling. The positive concordance rate was $44.4 \%(4 / 9)$ and $71.4 \%(5 / 7)$ in early-stage and advanced-stage NSCLC patients, respectively (Fig. 2a and c). Three patients (No.7, 14, 16) showed complete concordant genomic alterations in tumor tissue and cfDNA. Five patients (No.5, 8, 15, 18,
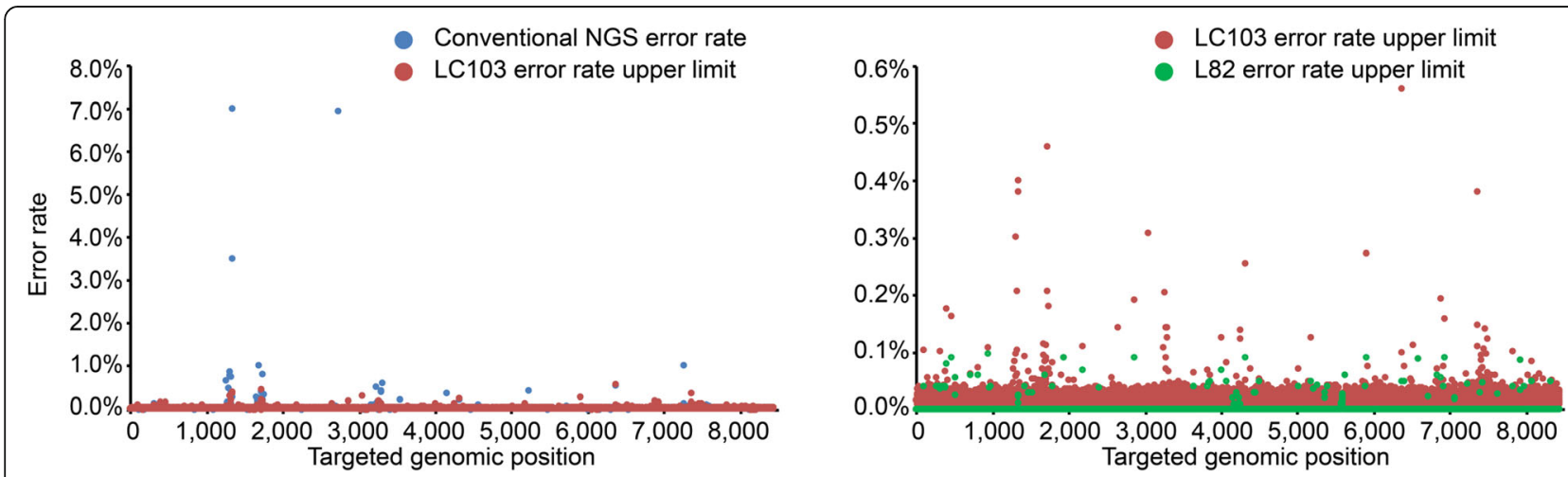

Fig. 1 Error rate reduction in LC103 and L82 gene panels compared to conventional NGS for Q30 bases. LC103 targets 103 regions of interest in 22 lung and colon cancer related genes. L82 interrogates 82 regions in 17 overlapping genes with LC103. Data were generated on Illumina NextSeq. Only the overlapping bases between two panels are plotted. At each base position, error rates are calculated by dividing the number of error alterations by the total base coverage using the data from cell line FFPE references after removing known mutations, and from healthy individuals analyzed for cfDNA analysis. The error rate of LC103 is well below 1\%, which allows reliable mutation detection above mutant allele frequency (MAF) of $2 \%$ in tumor tissue. For all mutations in the Multiplex Reference standards (FFPE DNA or sections, Horizon Discovery), the observed allele frequencies are consistent with the expected allele frequencies. In the L82 dataset (green), recurrent background errors (<0.1\% of error rate) are shown in the figure. These errors appear at non-hotspot positions and can be further reduced using a position-specific unbiased approach 


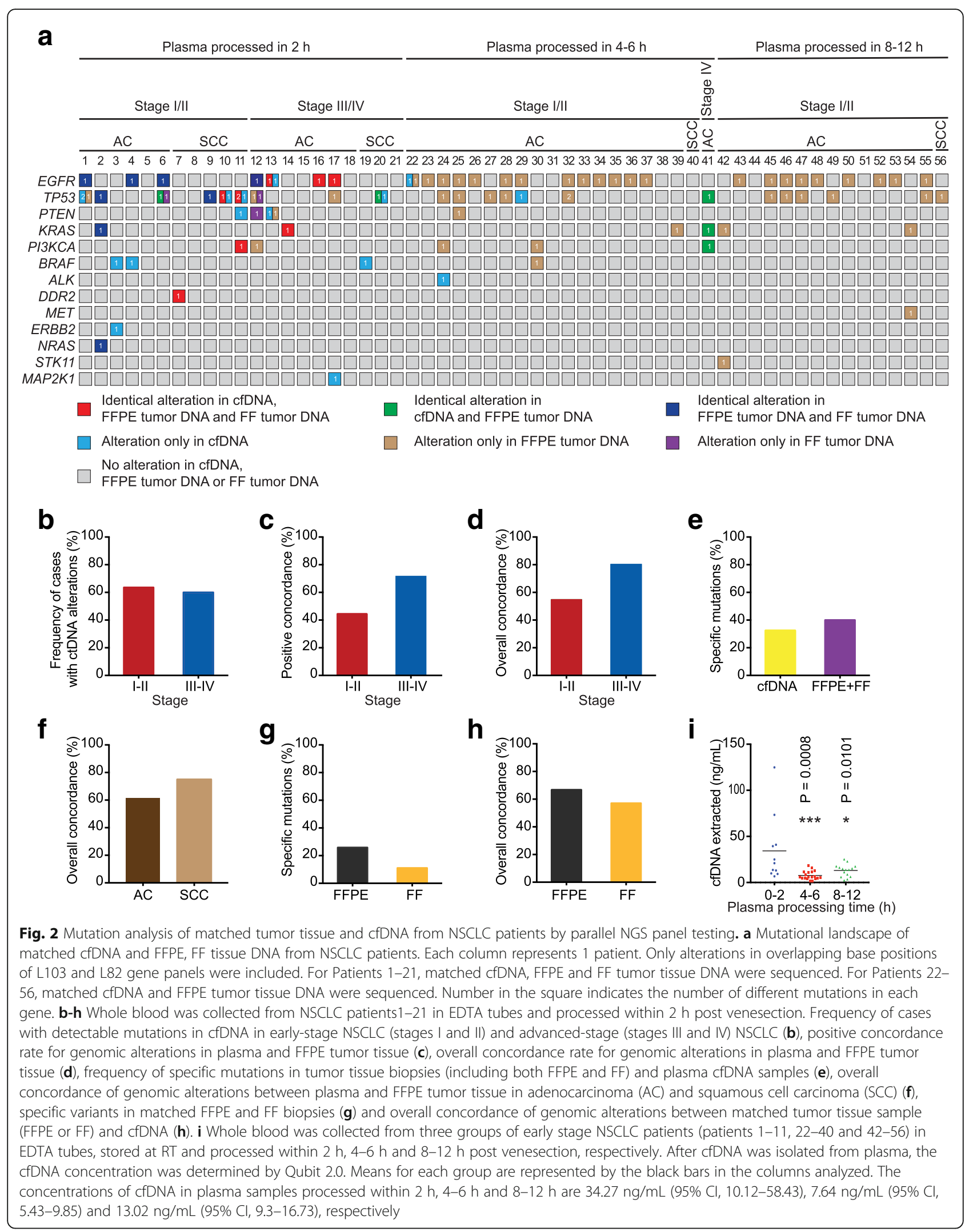


21) displayed negative concordance with genomic alterations detected in neither tumor tissue nor cfDNA. The overall concordance was $54.6 \%(6 / 11)$ in early-stage patients and $80 \%(8 / 10)$ in advanced-stage patients (Fig. 2a and d), consistent with recent findings [1]. Nevertheless, the discordance rate at the mutation level was high, similar to the reported results [7-9]. Only $22.5 \%(9 / 40)$ of somatic alterations were detected in all three sample types (FF, FFPE and cfDNA) and 2 additional concordant alterations were identified in both FFPE and cfDNA (Fig. 2a). In contrast, 32.5\% (13/40) and 40\% (16/40) mutations were cfDNA-specific and tissue-specific, respectively (Fig. 2a and e). Four high frequency mutations (15-51.03\%) in FFPE and FF tissues were not detected in cfDNA by both L82 panel sequencing and ddPCR (Additional file 4: Table S3). One high frequency mutation (TP53 c.481G > A) in FFPE and FF tissues were only detected in cfDNA by ddPCR but not by L82 panel sequencing, presumably because the ctDNA copies corresponding to the variant was extremely low in cfDNA. In addition, the overall concordance was $61.5 \%(2 / 6$ in early-stage and $6 / 7$ in advanced-stage) in lung adenocarcinoma patients and $75 \%$ (4/5 in early-stage and $2 / 3$ in advanced-stage) in squamous lung carcinoma patients (Fig. 2f). Collectively, genotyping FFPE tumor tissue and ctDNA in parallel on the same testing platform showed significant discordance in genomic alterations in tumor tissue and cfDNA, suggesting that intrinsic biological mechanisms might affect the concordance between tumor biopsy and ctDNA genomic profiling in different patients.

Previous studies have indicated that DNA damage in FFPE is a major source of erroneous identification of somatic variants with low to moderate (1 to $5 \%$ ) allelic frequencies [10]. This error has also been attributed to the discrepancies between tissue biopsy and ctDNA. Therefore, we collected FF tissue in a different region of each tumor from 21 patients and performed genotyping analyses on matched FFPE and FF samples. In a total of 27 tissue somatic variants, 63\% (17/27) are concordant between matched FF and FFPE samples. 25.9\% (7/27) and $11.1 \%(3 / 27)$ of somatic mutations are FFPE- and FF-specfic, respectively (Fig. 2a, g, and Additional file 5: Table S4). The overall concordance rate between matched FFPE sample/cfDNA and FF sample/cfDNA were $66.7 \%(14 / 21)$ and $57.1 \%(12 / 21)$, respectively, (Fig. $2 \mathrm{a}$ and $\mathrm{h}$ ). These results suggest that DNA damage in FFPE is not the major factor contributing to the discordance between tumor tissues and ctDNA in this study.

We next evaluated the impact of blood sample processing on mutation detection in cfDNA. Whole blood was collected from three groups of NSCLC patients in EDTA tubes by a single operator following the same protocol and then processed within $2 \mathrm{~h}, 4-6 \mathrm{~h}$ and 8$12 \mathrm{~h}$ post venesection. We found that cfDNA concentration in plasma processed within $2 \mathrm{~h}$ was significantly higher than that within $4-6 \mathrm{~h}$ or $8-12 \mathrm{~h}$ (Fig. 2i). The low cfDNA concentration at $4-6 \mathrm{~h}$ is most likely caused by cfDNA degradation, whereas the elevated cfDNA concentration at $8-12 \mathrm{~h}$ might be due to the genomic DNA released from leukocytes. The positive detection rate decreased significantly with the extended processing time (Fig. 2a). All 5 concordant cases had no genomic alterations detected in both tumor and plasma. Our results highlight the importance of processing blood samples in the EDTA tubes within $2 \mathrm{~h}$ to improve mutation detection sensitivity in cfDNA.

In summary, significant discordance in the genomic alterations of the matched tumor tissues and ctDNA was observed by genotyping tumor tissue and ctDNA in parallel using the same NGS panel platform. Many pre-analytical, analytical and biologic factors may affect the genotyping results on tissue and ctDNA in a clinical oncology setting. Among these factors, the intrinsic heterogeneous mutation pattern in tumor tissues and ctDNA can jeopardize the clinical benefit of precision medicine. Further understanding biologic factors that affect ctDNA release are needed. Improving the assay sensitivity and specificity of either genotyping approach alone is unlikely to resolve this discordant genotyping issue. To enhance assay accuracy and clinical utility, parallel NGS panel testing on multiple sample types for each patient may be warranted for effective guidance of cancer targeted therapies and possible early detection of cancer. International standards for tumor molecular profiling using tumor tissues and ctDNA should be established.

\section{Additional files}

Additional file 1: Table S1. Clinical characteristics of enrolled NSCLC patients. (XLSX $12 \mathrm{~kb}$ )

Additional file 2: Material and methods. (DOCX $29 \mathrm{~kb}$ )

Additional file 3: Table S2. Somatic alterations detected in cfDNA of NSCLC patients. (XLSX $13 \mathrm{~kb}$ )

Additional file 4: Table S3. Validation of ctDNA variants by ddPCR. (XLSX $10 \mathrm{~kb}$ )

Additional file 5: Table S4. Somatic alterations detected in tumor of NSCLC patients. (XLSX $15 \mathrm{~kb}$ )

\section{Abbreviations}

cfDNA: Cell-free DNA; ctDNA: Circulating tumor DNA; ddPCR: Droplet digital PCR; FF: Fresh frozen; FFPE: Formalin-fixed paraffin-embedded; MAF: Mutant allele frequency; NGS: Next-generation sequencing; NSCLC: Non-small-cell lung cancer

\section{Funding}

This work was supported by grants from the National Natural Science Foundation of China $(31525016,81672833)$, Personalized MedicinesMolecular Signature-based Drug Discovery and Development, the Strategic Priority Research Program of the Chinese Academy of Sciences 
(XDA12010101), the Ministry of Science and Technology of China (2014DFA33010), the CAS/SAFEA International Partnership Program for Creative Research Teams and Shanghai Hospital Development Center (SHDC22014011). The authors gratefully acknowledge the support of SA-SIBS scholarship program

\section{Availability of data and materials}

Please contact the corresponding author for all data requests.

\section{Authors' contributions}

JFC, JTL and GS designed the experiments. QMG, LW, XMH, and WJY collected clinical samples. JLW, QMG and JFX performed experiments and analyzed data. JLW, QMG, GS, JTL and JFC interpreted results. The manuscript was drafted by QMG, JLW, and edited by GS and JFC. All authors read and approved the final manuscript.

\section{Ethics approval and consent to participate}

All human samples are collected under an Institutional Review Board approved protocol (No.KS (P)1801).

\section{Consent for publication}

Written informed consent was obtained from the patients for publication of this letter.

\section{Competing interests}

The authors declare no competing financial interests.

\section{Publisher's Note}

Springer Nature remains neutral with regard to jurisdictional claims in published maps and institutional affiliations.

\section{Author details}

${ }^{1}$ Department of Laboratory Medicine, Shanghai Chest Hospital, Shanghai Jiao Tong University, Shanghai, China. ${ }^{2}$ State Key Laboratory of Cell Biology, CAS Center for Excellence in Molecular Cell Science, Shanghai Institute of Biochemistry and Cell Biology, Chinese Academy of Sciences, University of Chinese Academy of Sciences, 320 Yueyang Road, Shanghai 200031, China. ${ }^{3}$ Department of Research and Development, Shanghai Zhengu Biotech Ltd. Shanghai, China.

Received: 20 April 2018 Accepted: 9 August 2018

Published online: 28 August 2018

\section{References}

1. Phallen J, Sausen M, Adleff V, Leal A, Hruban C, White J, Anagnostou V, Fiksel J, Cristiano S, Papp E, et al. Direct detection of early-stage cancers using circulating tumor DNA. Sci Transl Med. 2017:9

2. Abbosh C, Birkbak NJ, Wilson GA, Jamal-Hanjani M, Constantin T, Salari R, Le Quesne J, Moore DA, Veeriah S, Rosenthal R, et al. Phylogenetic ctDNA analysis depicts early-stage lung cancer evolution. Nature. 2017;545:446-51.

3. Jamal-Hanjani M, Wilson GA, McGranahan N, Birkbak NJ, Watkins TBK, Veeriah S, Shafi S, Johnson DH, Mitter R, Rosenthal R, et al. Tracking the evolution of non-small-cell lung Cancer. N Engl J Med. 2017;376:2109-21.

4. Lanman RB, Mortimer SA, Zill OA, Sebisanovic D, Lopez R, Blau S, Collisson EA, Divers SG, Hoon DS, Kopetz ES, et al. Analytical and clinical validation of a digital sequencing panel for quantitative, Highly Accurate Evaluation of Cell-Free Circulating Tumor DNA. PLoS One. 2015;10:e0140712.

5. Frampton GM, Fichtenholtz A, Otto GA, Wang K, Downing SR, He J, SchnallLevin M, White J, Sanford EM, An P, et al. Development and validation of a clinical cancer genomic profiling test based on massively parallel DNA sequencing. Nat Biotechnol. 2013;31:1023-31.

6. Jones S, Anagnostou V, Lytle K, Parpart-Li S, Nesselbush M, Riley DR, Shukla M, Chesnick B, Kadan M, Papp E, et al. Personalized genomic analyses for cancer mutation discovery and interpretation. Sci Trans/ Med. 2015;7: 283ra253.

7. Kuderer NM, Burton KA, Blau S, Rose AL, Parker S, Lyman GH, Blau CA. Comparison of 2 commercially available next-generation sequencing platforms in oncology. JAMA Oncol. 2017;3:996-8.

8. Torga G, Pienta KJ. Patient-paired sample congruence between 2 commercial liquid biopsy tests. JAMA Oncol. 2017;
9. Chae YK, Davis AA, Carneiro BA, Chandra S, Mohindra N, Kalyan A, Kaplan J, Matsangou M, Pai S, Costa R, et al. Concordance between genomic alterations assessed by next-generation sequencing in tumor tissue or circulating cell-free DNA. Oncotarget. 2016;7:65364-73.

10. Chen L, Liu P, Evans TC Jr, Ettwiller LM. DNA damage is a pervasive cause of sequencing errors, directly confounding variant identification. Science. 2017; 355:752-6.

\section{Ready to submit your research? Choose BMC and benefit from:}

- fast, convenient online submission

- thorough peer review by experienced researchers in your field

- rapid publication on acceptance

- support for research data, including large and complex data types

- gold Open Access which fosters wider collaboration and increased citations

- maximum visibility for your research: over $100 \mathrm{M}$ website views per year

At $\mathrm{BMC}$, research is always in progress.

Learn more biomedcentral.com/submissions 